\title{
SYMPLECTIC STIEFEL HARMONICS AND HOLOMORPHIC REPRESENTATIONS OF SYMPLECTIC GROUPS
}

\section{BY}

TUONG TON-THAT

ABSTRACT. Let $I_{k}$ denote the identity matrix of order $k$ and set

$$
s_{k}=\left[\begin{array}{cc}
0 & -I_{k} \\
I_{k} & 0
\end{array}\right]
$$

Let $\mathrm{Sp}(k, \mathrm{C})$ denote the group of all complex $2 k \times k$ matrices which satisfy the equation $g s_{k} g^{t}=s_{k}$. Let $E$ be the linear space of all $n \times 2 k$ complex matrices with $k>n$, and let $S\left(E^{*}\right)$ denote the symmetric algebra of all complex-valued polynomial functions on $E$. The study of the action of $\mathrm{Sp}(k, \mathrm{C})$, which is obtained by right translation on $S\left(E^{*}\right)$, leads to a concrete and simple realization of all irreducible holomorphic representations of $\mathrm{Sp}(k, \mathrm{C})$. In connection with this realization, a theory of symplectic Stiefel harmonics is also established. This notion may be thought of as a generalization of the spherical harmonics for the symplectic Stiefel manifold.

Introduction. Let $I_{k}$ denote the unit matrix of order $k$ and set

$$
s_{k}=\left[\begin{array}{cc}
0 & -I_{k} \\
I_{k} & 0
\end{array}\right] \text {. }
$$

Let $\mathrm{Sp}(k, \mathrm{C})$ denote the group of all complex $2 k \times 2 k$ matrices $g$ satisfying $g s_{k} g^{t}=s_{k}$. If $G$ is a complex Lie group and $V$ is a finite-dimensional complex vector space, then by a holomorphic representation of $G$ in $V$ we mean a complex analytic homomorphism of $G$ into GL $(V)$. The object of this paper is to present, in as simple and explicit a form as possible, a description of all irreducible holomorphic representations (up to equivalence) of the complex linear symplectic group $\operatorname{Sp}(k, C)$. This realization is obtained by analyzing certain natural actions of $\mathrm{Sp}(k, \mathrm{C})$ on a symmetric algebra of polynomial functions of matrix argument. We rely on some general results of S. Helgason [3] and B. Kostant [4] to study this symmetric algebra. The techniques they used to decompose a symmetric algebra of polynomial functions were based on the existence of a symmetric bilinear form defined on this algebra. The situation is somewhat different in the context of our investigation, because

Received by the editors December 24, 1975 and, in revised form, March 16, 1976.

AMS (MOS) subject classifications (1970). Primary 22E45; Secondary 13F20.

Key words and phrases. Symmetric algebras of polynomial functions, irreducible holomorphic representations of symplectic groups, symplectic Stiefel harmonics. 
the bilinear form defined on our algebra, which is required to be invariant under the above action of $\operatorname{Sp}(k, \mathrm{C})$, is not symmetric. Thus modification and adaptation are necessary. This constitutes our main contribution in $\$ 1$.

Theorem 2.2 of $\$ 2$ is the central result of this paper. As a consequence of this theorem, we also obtain certain irreducible representations of the unitary symplectic group $\operatorname{Sp}(k)$ on a space of continuous functions defined on a symplectic Stiefel manifold. These functions, which we call symplectic Stiefel harmonics, are to the unitary symplectic group what the Stiefel harmonics are to the rotation group (cf. [2] and [6]). Since the Stiefel harmonics generalize the spherical harmonics, in view of the importance of this notion, the symplectic Stiefel harmonics seem interesting.

The main results of this paper were announced in [7]. Finally, it is a great pleasure for the author to express here his gratitude to the Department of Mathematics of Harvard University for the postdoctoral fellowship that supported him during the accomplishment of this work.

1. A decomposition of the symmetric algebra $S\left(E^{*}\right)$. Suppose $k$ and $n$ are integers with $k>n>2$. Set $G=\operatorname{Sp}(k, C)$ and let $E$ denote the vector space of all complex $n \times 2 k$ matrices. Then $G$ acts linearly on $E$ by right multiplication and leaves invariant the skew-symmetric bilinear form on $E$ given by

$$
(X, Y)=\operatorname{trace}\left(X s_{k} Y^{t}\right), \text { for all } X, Y \text { in } E .
$$

If $X \in E$, let $X^{*}$ denote the linear form $Y \rightarrow(X, Y)$ on $E$. Since $(\cdot, \cdot)$ is obviously nondegenerate, $E$ is isomorphic to its dual $E^{*}$ via the mapping $X \rightarrow X^{*}$. Consider the symmetric algebra $S\left(E^{*}\right)$ of all complex-valued polynomial functions on $E$, and define a representation $R$ of $G$ on $S\left(E^{*}\right)$ by $(R(g) p)(X)=p(X g)\left(p \in S\left(E^{*}\right), X \in E, g \in G\right)$. Given $X \in E$, let $X^{*}(D)$ denote the differential operator defined by

$$
\left(X^{*}(D) f\right)(Y)=\left\{\frac{d}{d t} f(Y+t X)\right\}_{t=0}, \quad\left(f \in S\left(E^{*}\right), t \in \mathbf{R}, X, Y \in E\right) .
$$

Define $\left(X_{1}^{*} \cdots X_{r}^{*}\right)(D) f=X_{1}^{*}(D)\left[\left(X_{2}^{*} \cdots X_{r}^{*}\right)(D) f\right]$ inductively on $r$. If $m$ and $l$ are nonnegative integers and if $\S_{m}$ denotes the symmetric group of $m$ elements, then a simple argument by induction on $l$ shows that

$$
\begin{aligned}
& {\left[X_{1}^{*} \cdots X_{l}^{*}(D)\right] Y_{1}^{*} \cdots Y_{m}^{*}} \\
& \quad\left\{\begin{array}{l}
0 \quad \text { if } m<l, \\
\frac{(-1)^{l}}{(m-l) !} \sum_{\sigma \in \delta_{m}} X_{1}^{*}\left(Y_{\sigma(1)}\right) \cdots X_{l}^{*}\left(Y_{\sigma(l)}\right) Y_{\sigma(l+1)}^{*} \cdots Y_{\sigma(m)}^{*} \text { if } m \geqslant l .
\end{array}\right.
\end{aligned}
$$


If follows immediately from the above equation and by linearity that the mapping $X^{*} \rightarrow X^{*}(D)(X \in E)$ can be extended to an algebraic homomorphism $p \rightarrow p(D)$ of $S\left(E^{*}\right)$ into the symmetric algebra $S(E)$ of differential operators on $E$. As direct consequences of equation (1.1), one obtains the following relations:

$$
\begin{gathered}
p(D)\left(Y^{*}\right)^{m}=\left((-1)^{l} m(m-1) \cdots(m-l+1) p(Y)\right)\left(Y^{*}\right)^{m-l} \\
p(D) q=(-1)^{l} q(D) p
\end{gathered}
$$

where $l$ and $m$ are integers with $m \geqslant l \geqslant 0$, and $p, q$ are homogeneous elements of $S\left(E^{*}\right)$ of degree $l$.

Define a bilinear form $\langle\cdot, \cdot\rangle$ on $S\left(E^{*}\right)$ by

$$
\left\langle f_{1}, f_{2}\right\rangle=\left(f_{1}(D) f_{2}\right)(0) \quad\left(f_{1}, f_{2} \in S\left(E^{*}\right)\right) .
$$

If $p$ and $q$ are homogeneous polynomials of degree $m$ and $l$, respectively, then one can easily deduce the following formulas from equations (1.1), (1.2), and (1.3):

$$
\begin{gathered}
\langle p, q\rangle= \begin{cases}(-1)^{l}\langle q, p\rangle & \text { if } l=m, \\
\langle q, p\rangle=0 & \text { if } l \neq m ;\end{cases} \\
\left\langle\frac{\left(X^{*}\right)^{m}}{m !}, p\right\rangle=p(X), \\
\left\langle X^{*}, Y^{*}\right\rangle=-(X, Y) .
\end{gathered}
$$

The fact that the mapping $p \rightarrow p(D)$ is an algebraic isomorphism is an immediate consequence of the following result.

LEMMa 1.1. The bilinear form $\langle\cdot, \cdot\rangle$ is nondegenerate.

Proof. We begin by observing that if $X \in E$ then $R(g) X^{*}=\left(X^{-1}\right)^{*}$ for all $g$ in $G$. If $M_{r t} \in E$ denotes the matrix with entry 1 in position $(r, t)$ and 0 everywhere, then

$$
\left[R\left(s_{k}\right) M_{u v}^{*}\right]\left(M_{i j}\right)=\left(M_{u v} s_{k}^{-1}\right)^{*}\left(M_{i j}\right)=\delta_{u i} \delta_{v j} \quad(1 \leqslant i, u \leqslant n ; 1 \leqslant j, v \leqslant 2 k) .
$$

It follows then from (1.1) that if $p=\Pi_{(1<i<n, 1<j<2 k)}\left(M_{i j}^{*}\right)^{\alpha_{i j}}$ and $q=$ $\Pi_{(1<i<n, 1<j<2 k)}\left(M_{i j}^{*}\right)^{\beta_{i j}}$ with $\Sigma_{i j} \alpha_{i j}=\Sigma_{i j} \beta_{i j}=m$, then

$$
\begin{aligned}
& \left\langle p, R\left(s_{k}\right) q\right\rangle=0 \quad \text { if } p \neq q, \\
& \left\langle p, R\left(s_{k}\right) p\right\rangle=\alpha_{11} ! \alpha_{12} ! \cdots ! \alpha_{n, 2 k} ! .
\end{aligned}
$$

Define a conjugation $X^{*} \rightarrow \tilde{X}^{*}$ on $E^{*}$ by setting $\tilde{X}^{*}=(\bar{X})^{*}$. This conjugation extends to a conjugation $p \rightarrow \tilde{p}$ on $S\left(E^{*}\right)$; moreover, we have $\widetilde{R(g)} p=$ $R(\bar{g}) \tilde{p}(g \in G)$. Let 


$$
\begin{array}{r}
f=\sum_{m_{i j}>0} c\left(m_{11}, \ldots, m_{n, 2 k}\right)\left(M_{11}^{*}\right)^{m_{11}}\left(M_{12}^{*}\right)^{m_{12}} \cdots\left(M_{n, 2 k}^{*}\right)^{m_{n, 2 k}} \\
\left(c\left(m_{11}, \ldots, m_{n, 2 k}\right) \in \mathbf{C}\right)
\end{array}
$$

be a homogeneous polynomial of degree $m=\Sigma_{i j} m_{i j}$; then from relation (1.7) we infer that

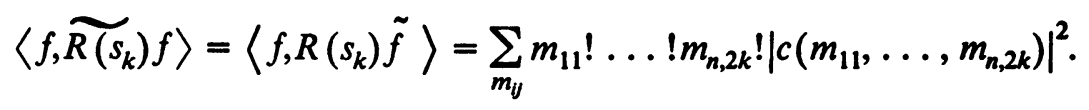

This relation in turn shows that $\langle\cdot, \cdot\rangle$ is a nondegenerate bilinear form on $S\left(E^{*}\right)$.

Definition 1.2. A polynomial $f \in S\left(E^{*}\right)$ is said to be $G$-invariant if $R(g) f=f$ for all $g \in G$. A differential operator $p(D) \in S(E)$ will be called $G$-invariant if

$$
R(g)(p(D) f)=p(D)(R(g) f) \quad\left(g \in G, f \in S\left(E^{*}\right)\right)
$$

The following result will be needed later.

Proposition 1.3. A polynomial $p \in S\left(E^{*}\right)$ is $G$-invariant if and only if the differential operator $p(D)$ is $G$-invariant.

Proof. Before proving this proposition, a preliminary observation is needed, namely: if $p$ and $f$ are elements of $S\left(E^{*}\right)$ then $\left[R(g) p(D) R\left(g^{-1}\right)\right] f$ $=[(R(g) p)(D)] f$ for all $g \in G$. To see this, we notice that it is sufficient to establish this identity for $p=X_{1}^{*} X_{2}^{*} \ldots X_{l}^{*}$ and $f=Y_{1}^{*} Y_{2}^{*} \ldots Y_{m}^{*}\left(X_{i}, Y_{j} \in\right.$ $E ; m>l)$. Now, for all $g \in G$ and $Z \in E$, we have

$$
\left(R(g) p(D) R\left(g^{-1}\right) f\right)(Z)=\left(p(D) R\left(g^{-1}\right) f\right)(Z g)
$$

But clearly for such $p$ and $f, R(g) p=\left(X_{1} g^{-1}\right)^{*} \ldots\left(X_{1} g^{-1}\right)^{*}$ and $R\left(g^{-1}\right) f$ $=\left(Y_{1} g\right)^{*} \ldots\left(Y_{m} g\right)^{*}$. Therefore, according to equation (1.1),

$$
\begin{aligned}
\left(p(D) R\left(g^{-1}\right) f\right)(Z g) \\
=\frac{(-1)^{l}}{(m-l) !} \sum_{\sigma \in \delta_{m}} X_{1}^{*}\left(Y_{\sigma(1)} g\right) \ldots X_{l}^{*}\left(Y_{\sigma(l)} g\right)\left(Y_{\sigma(l+1)} g\right)^{*}(Z g) \\
\quad \ldots\left(Y_{\sigma(m)} g\right)^{*}(Z g) \\
=\frac{(-1)^{l}}{(m-l) !} \sum_{\sigma \in \delta_{m}}\left(X_{1} g^{-1}\right)^{*}\left(Y_{\sigma(1)}\right) \\
\quad \ldots\left(X_{l} g^{-1}\right)^{*}\left(Y_{\sigma(l)}\right) Y_{\sigma(l+1)}^{*}(Z) \ldots Y_{\sigma(m)}^{*}(Z) \\
\quad[(R(g) p)(D) f](Z) .
\end{aligned}
$$


Hence, $\left[R(g) p(D) R\left(g^{-1}\right)\right] f=[[R(g) p](D)] f$. Now if $p$ is $G$-invariant, then $[(R(g) p)(D)] f=p(D) f$ for all $g \in G$ and $f \in S\left(E^{*}\right)$. By the preceding paragraph, we infer that $\left[R(g) p(D) R\left(g^{-1}\right)\right] f=p(D) f$, which is obviously equivalent to saying that $p(D)$ is $G$-invariant. Conversely, if $p(D)$ is $G$-invariant, then $(R(g) p)(D)=p(D)$ for all $g \in G$. But since the mapping $p \rightarrow p(D)$ is injective this implies that $R(g) p=p$ for all $g \in G$. This achieves the proof of the proposition.

Definition 1.4. A polynomial $f \in S\left(E^{*}\right)$ is called $G$-harmonic if it is annihilated by all homogeneous $G$-invariant differential operators with constant coefficients and without constant term.

Now, if $J\left(E^{*}\right)$ denotes the subalgebra of $S\left(E^{*}\right)$ consisting of all $G$-invariant polynomials, and if $J^{+}\left(E^{*}\right)$ denotes the set of all $G$-invariant polynomials without constant term, then it follows from Proposition 1.3 that $f$ is $G$-harmonic if and only if $p(D) f=0$ for all $p \in J^{+}\left(E^{*}\right)$. But from the theory of polynomial invariants (cf. [8, Chapter IV]), $J\left(E^{*}\right)$ is generated by the constant function 1 and $\frac{1}{2} n(n-1)$ algebraically independent homogeneous polynomials $p_{i j}$ which are defined by

$$
\begin{array}{r}
p_{i j}(X)=\sum_{l=1, \ldots, k}\left(X_{i, l+k} X_{j l}-X_{i l} X_{j, l+k}\right) \\
\quad\left(1 \leqslant i<j \leqslant n ; X=\left(X_{r s}\right) \in E\right) .
\end{array}
$$

If follows that, if $H\left(E^{*}\right)$ denotes the subspace of $S\left(E^{*}\right)$ consisting of all $G$-harmonic polynomials, then $H\left(E^{*}\right)=\left\{f \in S\left(E^{*}\right): p_{i j}(D) f=0,1<i<\right.$ $j<n\}$.

Let $J^{+}\left(E^{*}\right) S\left(E^{*}\right)$ be the ideal in $S\left(E^{*}\right)$ generated by $J^{+}\left(E^{*}\right)$, and denote by $V$ the algebraic variety in $E$ of common zeros of polynomials in $J^{+}\left(E^{*}\right) S\left(E^{*}\right)$. Then from the above paragraph, it is easy to see that $V=\{x$ $\left.\in E: X s_{k} X^{t}=0\right\}$.

THEOREM 1.5. The ideal $J^{+}\left(E^{*}\right) S\left(E^{*}\right)$ is prime.

In the proof we use several lemmas.

LEMMA 1.6. Let $W$ be a $2 k$-dimensional vector space over a subfield of the complex numbers, and let $(\cdot \mid \cdot)$ be a nondegenerate skew-symmetric bilinear form on $W$. Suppose $U$ is a subspace of $W$ of dimension $n(n<k)$ such that the restriction of $(\cdot \mid \cdot)$ to $U$ is the zero form; then there exist $k$ pairs of vectors $\left(\alpha_{1}, \beta_{1}\right),\left(\alpha_{2}, \beta_{2}\right), \ldots,\left(\alpha_{k}, \beta_{k}\right)$ with the following properties:

(1) The vectors $\alpha_{1}, \ldots, \alpha_{n}$ form a basis for $U$.

(2) $\left(\alpha_{i}, \beta_{j}\right)=\delta_{i j}, 1<i, j<k$.

(3) $\left(\alpha_{i}, \alpha_{j}\right)=\left(\beta_{i}, \beta_{j}\right)=0,1 \leqslant i, j \leqslant k$.

For a proof of this well-known fact see, for example, E. Artin's Geometric 
algebra, Interscience (Theorem 3.8, p. 120).

LEMMA 1.7. Let 1 denote the matrix $\left[I_{n} 0\right]$ in $E$, where $I_{n}$ is the identity matrix of order $n$ and 0 is the $n \times(2 k-n)$ zero matrix. If $V_{n}$ denotes the subset of $V$ consisting of all elements of maximal rank in $V$, then $V_{n}$ is the orbit $\operatorname{Orb}(1)$ of 1 under the action of $G$.

Proof. It is obvious that $\operatorname{Orb}(1)$ is contained in $V_{n}$. To establish the reverse inclusion we consider the vector space $\mathbf{C}^{1 \times 2 k}$ and equip $\mathbf{C}^{1 \times 2 k}$ with the bilinear form $(\cdot \mid \cdot)$ defined by

$$
(x \mid y)=\operatorname{tr}\left(x s_{k} y^{t}\right)=\sum_{i=1}^{k}\left(x_{i+k} y_{i}-x_{i} y_{i+k}\right) \quad\left(x, y \in \mathbf{C}^{1 \times 2 k}\right) .
$$

Clearly $(\cdot \mid \cdot)$ is skew-symmetric and nondegenerate. If $X \in E$ and $x_{j}$ denotes the $j$ th row of $X(1<j \leqslant n)$, we let $\operatorname{Span}\left\{x_{1}, \ldots, x_{n}\right\}$ be denoted by $U_{X}$. Now $X$ belonging to $V_{n}$ implies that $\operatorname{dim}\left(U_{X}\right)=n$ and that the restriction of $(\cdot \mid \cdot)$ to $U_{X}$ is the zero form. Thus the hypotheses of Lemma 1.6 are satisfied and we conclude that there exist vectors $z_{1}, \ldots, z_{n}, \alpha_{n+1}, \ldots, \alpha_{k}$ and $\beta_{n+1}, \ldots, \beta_{k}$ in $\mathbf{C}^{1 \times 2 k}$ such that the pairs $\left(x_{1}, z_{1}\right), \ldots,\left(x_{n}, z_{n}\right)$, $\left(\alpha_{n+1}, \beta_{n+1}\right), \ldots,\left(\alpha_{k}, \beta_{k}\right)$ verify conditions (1), (2), and (3) of Lemma 1.6. If $g_{1}$ denotes the $2 k \times 2 k$ matrix with rows arranged in the following order: $z_{1}, \ldots, z_{n}, \beta_{n+1}, \ldots, \beta_{k}, x_{1}, \ldots, x_{n}, \alpha_{n+1}, \ldots, \alpha_{k}$, then $g_{1}$ belongs to $G$. Set $g=s_{k} g_{1}^{t}$, then $g$ belongs to $G$ since $s_{k}$ and $g_{1}^{t}$ belong to $G$. Obviously, $X g=X s_{k} g_{1}^{t}=1$, i.e., $X \in \operatorname{Orb}(1)$. This achieves the proof of the lemma.

LEMma 1.8. Let $p_{i j}(1 \leqslant i<j \leqslant n)$ be the generators of $J^{+}\left(E^{*}\right) S\left(E^{*}\right)$ as defined by equation (1.8). Suppose $X \in E$ and $\operatorname{rank}(X)>n-1$, then the rank of the Jacobian matrix $J\left(p_{i j}\right)(X)=\left[D_{r s} p_{i j}\right](X)$ is equal to $\frac{1}{2} n(n-1)(1<r<$ $n ; 1<s<2 k$ ).

Proof. We proceed by induction on $n$. If $n=2$, the assertion is obviously true. Assume the lemma true for all $l \leqslant n-1$, and let $X \in E$ with $\operatorname{rank}(X)$ $\geqslant n-1$. We may assume that the $(n-1) \times 2 k$ matrix $X_{q}$ which is obtained by deleting the $q$ th row from $X$ has rank $n-1$. Next, let $X$ denote the matrix which is obtained from $X$ by shifting the $q$ th row of $X$ to the last position without changing the relative order of the other rows. Similarly, we order the polynomials $p_{i j}(1<i<j \leqslant n)$ by shifting the $n-1$ elements $p_{1 q}, \ldots, p_{q-1, q}, p_{q, q+1}, \ldots, p_{q n}$ in the last positions. Clearly, the Jacobian matrix $J\left(p_{12}, \ldots, p_{1 q}, \ldots, p_{q n}\right)(\tilde{X})$ has the same rank as $J\left(p_{i j}\right)(X)$. By a simple computation, we obtain

$$
J\left(p_{12}, \ldots, p_{q n}\right)(\tilde{X})=\left[\begin{array}{ll}
A & 0 \\
B & C
\end{array}\right]
$$

where $A=\left[D_{u v} p_{i j}\right]\left(X_{q}\right)(1 \leqslant i<j \leqslant n-1)$ and 


$$
C=\left[\begin{array}{cccccc}
x_{1, k+1} & \cdots & x_{1,2 k} & -x_{11} & \ldots & -x_{1, k} \\
\vdots & & \vdots & \vdots & & \\
x_{q-1, k+1} & \cdots & x_{q-1,2 k} & -x_{q-1,1} & \ldots & -x_{q-1, k} \\
-x_{q+1, k+1} & \cdots & -x_{q+1,2 k} & x_{q+1,1} & \ldots & x_{q+1, k} \\
\vdots & & & \vdots & & \\
-x_{n}, k+1 & \cdots & -x_{n, 2 k} & x_{n, 1} & \ldots & x_{n, k}
\end{array}\right]
$$

It is easy to see that $\operatorname{rank}(C)=\operatorname{rank}\left(X_{q}\right)=n-1$. Since by the inductive hypothesis $\operatorname{rank}(A)=\frac{1}{2}(n-2)(n-1)$, we conclude that

$$
\operatorname{rank}\left(J\left(p_{11}, \ldots, p_{q, n}\right)(\tilde{X})\right)=\operatorname{rank}(A)+\operatorname{rank}(C)=\frac{1}{2}(n-1) n .
$$

Lemma 1.9 (Kostant). Assume $J\left(E^{*}\right)$, as an algebra, is generated by $l$ algebraically independent homogeneous polynomials $p_{i}(1 \leqslant i \leqslant l)$ and by the constant functions. Assume also that there exists an orbit $\mathcal{O}$ (under the action of $G)$ that is dense in $V$. Then $V$ is a subvariety of dimension $2 n k-l$. Moreover, $J^{+}\left(E^{*}\right) S\left(E^{*}\right)$ is prime if and only if there exists $X \in V$ such that $\left(d p_{i}\right)_{X}$, $1<i \leqslant l$, are linearly independent.

\section{Proof. See [4, Proposition 7, p. 347].}

Proof OF THEOREM 1.5. The proof follows immediately from Lemmas 1.7, 1.8 , and 1.9 if we observe that $V_{n}$ is obviously dense in $V$.

Suppose $X \in V$ and $m$ is any nonnegative integer; then we notice that $p_{i j}(D)\left(X^{*}\right)^{m}=0$ for all $i, j(1 \leqslant i<j \leqslant n)$, so that $\left(X^{*}\right)^{m}$ is $G$-harmonic. Indeed, this fact is obvious for $m<2$ and it is an immediate consequence of equation (1.2) for $m \geqslant 2$. Let $H_{V}\left(E^{*}\right)$ denote the subspace of $H\left(E^{*}\right)$ spanned by all $\left(X^{*}\right)^{m}(X \in V, m \geqslant 0)$. If $A$ is a subspace of $S\left(E^{*}\right)$ and $m$ is a nonnegative integer, $A_{m}$ shall denote the set of elements in $A$ of degree $m ; A$ is said to be homogeneous if $A=\Sigma_{m>0} A_{m}$. The spaces $H\left(E^{*}\right), H_{V}\left(E^{*}\right)$, $J\left(E^{*}\right)$, and the ideal $J^{+}\left(E^{*}\right) S\left(E^{*}\right)$ are clearly homogeneous.

Under the above notations we obtain the following result.

THEOREM 1.10. The space $S\left(E^{*}\right)$ is decomposed into a direct sum as $S\left(E^{*}\right)$ $=J^{+}\left(E^{*}\right) S\left(E^{*}\right) \oplus H\left(E^{*}\right)$. In addition, the identities $S\left(E^{*}\right)=J\left(E^{*}\right) \otimes$ $H\left(E^{*}\right)$ and $H\left(E^{*}\right)=H_{V}\left(E^{*}\right)$ also hold.

Proof. Since all subspaces involved are homogeneous, we need only to prove that, for $m \geqslant 2$,

$$
\left(S\left(E^{*}\right)\right)_{m}=\left(J^{+}\left(E^{*}\right) S\left(E^{*}\right)\right)_{m} \oplus\left(H\left(E^{*}\right)\right)_{m}
$$

and 


$$
\left(H\left(E^{*}\right)\right)_{m}=\left(H_{V}\left(E^{*}\right)\right)_{m} .
$$

Recall from (1.4) that $\langle p, q\rangle=(-1)^{m}\langle q, p\rangle$ for all $p, q \in\left(S\left(E^{*}\right)\right)_{m}$. Let $\langle\cdot, \cdot\rangle_{m}$ denote the restriction of the bilinear form $\langle\cdot, \cdot\rangle$ to $\left(S\left(E^{*}\right)\right)_{m}$; then $\langle\cdot, \cdot\rangle_{m}$ is either symmetric or skew-symmetric depending on the parity of $m$. But in either case, by Lemma $1.1,\langle\cdot, \cdot\rangle_{m}$ is nondegenerate; thus if $A$ is a subspace of $\left(S\left(E^{*}\right)\right)_{m}$ we shall denote by $A^{\perp}$ the subspace of $\left(S\left(E^{*}\right)\right)_{m}$ which is orthogonal to $A$ with respect to $\langle\cdot, \cdot\rangle_{m}$. We contend that $\left(J^{+}\left(E^{*}\right) S\left(E^{*}\right)\right)_{m}^{\perp}=\left(H\left(E^{*}\right)\right)_{m}$. Indeed, if $h \in\left(H\left(E^{*}\right)\right)_{m}$ and if $\in$ $\left(J^{+}\left(E^{*}\right) S\left(E^{*}\right)\right)_{m}$ where $j \in J^{+}\left(E^{*}\right)$, then

$$
\langle j f, h\rangle_{m}=[(j f)(D) h](0)=[f(D)(j(D) h)](0)=0 .
$$

Conversely, if $h \in\left(J^{+}\left(E^{*}\right) S\left(E^{*}\right)\right)_{m}^{\perp}$, then in particular we must have $\left\langle p_{i j} f, h\right\rangle_{m}=0$ for all generators $p_{i j}$ of $J^{+}\left(E^{*}\right) S\left(E^{*}\right)$. But then, we have

$$
0=\left\langle p_{i j} f, h\right\rangle_{m}=\left[p_{i j}(D) f(D) h\right](0)=\left\langle f, p_{i j}(D) h\right\rangle_{m-2} .
$$

Since $\langle\cdot, \cdot\rangle_{m-2}$ is nondegenerate, $p_{i j}(D) h$ must vanish everywhere, i.e., $h \in$ $\left(H\left(E^{*}\right)\right)_{m}$. To show that $\left(S\left(E^{*}\right)\right)_{m}$ is an orthogonal direct sum of $\left(J^{+}\left(E^{*}\right) S\left(E^{*}\right)\right)_{m}$ and $\left(H\left(E^{*}\right)\right)_{m}$, it is now sufficient to show that $\langle\cdot, \cdot\rangle_{m}$ is nondegenerate on $\left(J^{+}\left(E_{\tilde{N}}^{*}\right) S\left(E^{*}\right)\right)_{m}$. So let jf $\in\left(J^{+}\left(E^{*}\right) S\left(E^{*}\right)\right)_{m}$; then since

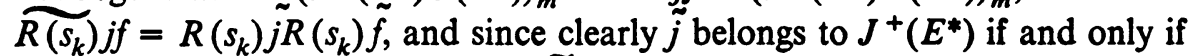
$j \in J^{+}\left(E^{*}\right)$, it follows that $R\left(s_{k}\right) \tilde{j f}$ belongs to $\left(\left(J^{+}\left(E^{*}\right) S\left(E^{*}\right)\right)_{m}\right.$. Now, by Lemma 1.1, the quantity $\left\langle j f, R\left(s_{k}\right) \tilde{j f}\right\rangle$ is strictly positive if $j f$ is different from zero.

Now suppose that $f \in\left(H\left(E^{*}\right)\right)_{m}$ satisfies $\left\langle f,\left(X^{*}\right)^{m}\right\rangle_{m}=0$ for all $X \in V$; then equation (1.5) implies that $f(X)=0$ for all $X \in V$. By Hilbert's Nullstellensatz, $f$ must belong to the nil radical of $J^{+}\left(E^{*}\right) S\left(E^{*}\right)$ which is obviously equal to the ideal $J^{+}\left(E^{*}\right) S\left(E^{*}\right)$ itself by Theorem 1.5 . Therefore, $f=0$ by the first part of the proof. From this, we infer that $\left(H\left(E^{*}\right)\right)_{m}=$ $\left(H_{V}\left(E^{*}\right)\right)_{m}$. To finish the proof of the theorem, we need only to invoke the following proposition proved by Kostant in [4].

Proposition 1.11. Assume that $\mathrm{J}^{+}\left(E^{*}\right) S\left(E^{*}\right)$ is prime and there exists an orbit $\mathcal{\theta}$ under $G$ such that $\mathcal{O}$ is dense in $V$. If $S\left(E^{*}\right)=J^{+}\left(E^{*}\right) S\left(E^{*}\right) \oplus$ $H\left(E^{*}\right)$ is a direct sum then the mapping $J\left(E^{*}\right) \otimes H\left(E^{*}\right) \rightarrow S\left(E^{*}\right)$ given by $f \otimes g \rightarrow f g$ is an isomorphism.

COROLlaRY 1.12. If $S(V)$ denotes the algebra of functions on $V$ obtained by restricting elements of $S\left(E^{*}\right)$ to $V$, then the restriction mapping $f \rightarrow f \mid V$ $\left(f \in H\left(E^{*}\right)\right)$ is a G-module isomorphism of $H\left(E^{*}\right)$ onto $S(V)$.

Proof. Since $V$ is the variety of the ideal $J^{+}\left(E^{*}\right) S\left(E^{*}\right)$ it follows readily from Theorem 1.10 that the map $f \rightarrow f \mid V\left(f \in H\left(E^{*}\right)\right)$ is a $G$-module epimorphism. Next, suppose that $f \in H\left(E^{*}\right)$ and $f(X)=0$ for all $X \in V$; 
then by Hilbert's Nullstellensatz $f \in J^{+}\left(E^{*}\right) S\left(E^{*}\right)$ since the latter is equal to its nil radical. But then Theorem 1.10 implies that $f=0$. This completes the proof of the corollary.

2. The irreducible holomorphic representations of $G$ and the symplectic Stiefel harmonics. In this section we shall make use of the Borel-Weil theory. Accordingly, we shall recall succinctly some results of this theory when the symplectic group $G$ is concerned. For the general theory, see [1] and [9].

Define

$$
\begin{aligned}
& C=\left\{\left[\begin{array}{cc}
c_{1} & 0 \\
0 & c_{1}^{\circ-1}
\end{array}\right] \in \mathrm{GL}(2 k, \mathbf{C}): c_{1} \text { diagonal } k \times k \text { matrix }\right\} \\
& U=\left\{\left[\begin{array}{cc}
u_{1} & 0 \\
u_{2} & u_{1}^{\vee}
\end{array}\right] \in \mathrm{GL}(2 k, \mathbf{C}): u_{1}^{\vee}=\left(u_{1}^{t}\right)^{-1}, u_{1} u_{2}^{t}-u_{2} u_{1}^{t}=0,\right. \\
& \left.u_{1} \text { lower triangular unipotent }\right\}, \\
& V=U^{t}=\left\{v \in \mathrm{GL}(2 k, \mathbf{C}): v=u^{t}, u \in U\right\} ;
\end{aligned}
$$

then $C, U$, and $V$ are closed subgroups of $G$. In fact, if we let $r \in \operatorname{GL}(k, C)$ and $\theta \in \mathrm{GL}(2 k, \mathrm{C})$ be given by

$$
r=\left[\begin{array}{lll}
0 & & .1 \\
& 1 & \\
1 & & 0
\end{array}\right] \text { and } \theta=\left[\begin{array}{cc}
I_{k} & 0 \\
0 & r
\end{array}\right] \text {, }
$$

then under the mapping $g \rightarrow \theta g \theta^{-1}(g \in G)$ the triple $\{C, U, V\}$ corresponds to the Gauss decomposition of $G$ as given in [9]. Let $T$ be a finite-dimensional irreducible holomorphic representation of $G$ in a complex vector space $H$. A nonzero vector $h \in H$ is called a weight vector corresponding to the weight $\lambda$ if $\lambda$ is a holomorphic character of the group $C$ and if $T$ satisfies the relation $T(c) h=\lambda(c) h$ for all $c \in C$. In this context, the highest weight vector of $T$ may be defined as the unique vector $h$ (up to a multiplicative constant factor) of $H$ such that $T(v) h=h$ for all $v \in V$. A character $\lambda$ of $G$ is called inductive if it is the highest weight of an irreducible holomorphic representation of $G$. According to [9], we know that if $\lambda$ is an inductive character of an irreducible holomorphic representation $T$ of $G$ then $\lambda$ is given by $c \rightarrow \lambda(c)=c_{11}^{m_{1}} c_{22}^{m_{2}} \cdots c_{k k}^{m_{k}}$, where the $k$-tuple $\left(m_{1}, \ldots, m_{k}\right)$ consists of integers satisfying the condition $m_{1}>m_{2}>\cdots>m_{k}>0$. Under this condition, the $k$-tuple $\left(m_{1}, \ldots, m_{k}\right)$ is called the signature of the representation $T$. 
Now let $B$ denote the lower triangular subgroup of $\operatorname{GL}(n, \mathbf{C})$ and define a holomorphic character $\xi=\xi\left(m_{1}, \ldots, m_{n}\right)$ on $B$ by setting $\xi(b)=$ $b_{11}^{m_{1}} b_{22}^{m_{2}} \cdots b_{n n}^{m_{n}}(b \in B)$, where the $m_{i}$ 's $(1 \leqslant i \leqslant n)$ are integers satisfying $m_{1} \geqslant m_{2} \geqslant \cdots \geqslant m_{n} \geqslant 0$. A polynomial $f \in S\left(E^{*}\right)$ will be called $\xi$-covariant if $f(b X)=\xi(b) f(X)(b \in B, X \in E)$. Let $H(E, \xi)$ denote the subspace of $H\left(E^{*}\right)$ constituted by all $\xi$-covariant $G$-harmonic polynomials. Notice that $H(E, \xi)$ is nonzero since if we let $f_{\xi} \in S\left(E^{*}\right)$ be defined by

$$
f_{\xi}(X)=\Delta_{1}^{m_{1}-m_{2}}(X) \Delta_{2}^{m_{2}-m_{3}}(X) \cdots \Delta_{n-1}^{m_{n-1}}-m_{n}(X) \Delta_{n}^{m_{n}}(X)
$$

where the $\Delta_{i}(X)(1 \leqslant i \leqslant n)$ are the principal minors of $X$, then it is easy to verify that $f_{\xi}$ belongs to $H(E, \xi)$. Furthermore, $H(E, \xi)$ is certainly invariant under $R$, because by definition the action of the $G$-invariant differential operators commutes with the action of $G$ on $S\left(E^{*}\right)$. Thus if we let $R(\cdot, \xi)$ denote the restriction of $R$ to $H(E, \xi)$ then under the above context we have the following result.

THEOREM 2.1. The representation $R(\cdot, \xi)$ of $G$ on $H(E, \xi)$ is an irreducible holomorphic representation of $G$, and its signature is $\left(m_{1}, m_{2}, \ldots, m_{n}, 0, \ldots, 0\right)$ ( $k$ factors).

Proof. Suppose $C, U, V$ are defined as in equation (2.1), then $C U$ is. a Borel subgroup of $G$. Define a holomorphic character $\zeta$ on $C U$ by setting $\zeta(c u)=$ $c_{11}^{m_{1}} \ldots c_{n n}^{m_{n}}(c u \in C U)$, with the same integers $m_{i}$ as in $\xi\left(m_{1}, \ldots, m_{n}\right)$. Let $\operatorname{Hol}(G, \zeta)$ denote the space of all $\zeta$-covariant holomorphic functions $f$ on $G$ (i.e., $f(c u g)=\zeta(c u) f(g)$, all $g \in G)$. Then by the Borel-Weil theorem the representation $\pi(\cdot, \zeta)$ of $G$ which is obtained by right translation on $\operatorname{Hol}(G, \zeta)$ is irreducible and its signature is $\left(m_{1}, \ldots, m_{n}, 0, \ldots, 0\right)$ (see [1], and also [9, Chapter XVI]). Define a map $\Phi$ from $H(E, \xi)$ into $\operatorname{Hol}(G, \zeta)$ by the equation $(\Phi f)(g)=f(1 g)(f \in H(E, \xi), g \in G)$, where $1=\left[I_{n} 0\right]$ is such that the orbit of 1 under the action of $G$ is a dense submanifold of $V$ (cf. Lemma 1.7). It is then routine to verify that $\Phi$ is a well-defined linear map which intertwines $R(\cdot, \xi)$ and $\pi(\cdot, \zeta)$. Moreover, $\Phi\left(f_{\xi}\right)$ is the highest weight vector of $\pi(\cdot, \zeta)$. Next, suppose $f \in H(E, \xi)$ is such that $\Phi f=0$, i.e., $f(1 g)=0$ for all $g \in G$. Then Corollary 1.12 together with Lemma 1.7 imply that $f=0$. It follows that $\Phi$ is a monomorphism; but since $\pi(\cdot, \zeta)$ is irreducible, $\Phi$ is a $G$-module isomorphism.

When $k=n$, the following theorem is an immediate consequence of Theorem 2.1.

TheOREM 2.2. Suppose that $E=\mathbf{C}^{k \times 2 k}(k \geqslant 2)$ and $\xi=\xi\left(m_{1}, \ldots, m_{k}\right)$; then the representations $R(\cdot, \xi)$ of $G$ on various spaces $H(E, \xi)$ realize up to equivalence all irreducible holomorphic representations of $G$ when the $m_{i}$ 's 
$(1<i<k)$ are allowed to take all integral values subject to the condition $m_{1} \geqslant m_{2} \geqslant \ldots \geqslant m_{k} \geqslant 0$. Moreover, to each representation $R(\cdot, \xi)$ corresponds a highest weight vector $f_{\xi} \in S\left(E^{*}\right)$ defined by the equation

$$
f_{\xi}(X)=\Delta_{1}^{m_{1}-m_{2}}(X) \Delta_{2}^{m_{2}-m_{3}}(X) \cdots \Delta_{k-1}^{m_{k-1}-m_{k}}(X) \Delta_{k}^{m_{k}}(X) \quad(X \in E)
$$

where the $\Delta_{i}(X)(1 \leqslant i \leqslant k)$ are the principal minors of $X$.

Assume $k>n \geqslant 2$ and let $G_{0}=G \cap U(2 k)$ denote the unitary linear symplectic group $\operatorname{Sp}(k)$. Let $K_{0}$ denote the image of $\operatorname{Sp}(k-n)$ under the imbedding

$$
\left[\begin{array}{ll}
u_{1} & u_{2} \\
u_{3} & u_{4}
\end{array}\right] \hookrightarrow\left[\begin{array}{cccc}
1 & 0 & 0 & 0 \\
0 & u_{1} & 0 & u_{2} \\
0 & 0 & 1 & 0 \\
0 & u_{3} & 0 & u_{4}
\end{array}\right] \quad\left(\left[\begin{array}{ll}
u_{1} & u_{2} \\
u_{3} & u_{4}
\end{array}\right] \in \operatorname{Sp}(k-n)\right) \text { of } \operatorname{Sp}(k-n)
$$

into $G_{0}$. Under the action of $G_{0}$ on $E$ it is obvious that the isotropy subgroup of $G_{0}$ at $1=\left[I_{n} 0\right]$ is $K_{0}$. Therefore, the orbit $\theta_{G_{0}}(1)$ of 1 under the action of $G_{0}$ is diffeomorphic to $K_{0} \backslash G_{0}$. We contend that $\theta_{G_{0}}(1)$ consists of all elements $X$ of the variety $V$ that satisfy $X X^{*}=I_{n}$. Indeed, it is clear that $\theta_{G_{0}}(1) \subset \operatorname{Orb}(1) \subset V$, and also $(1 g)(1 g)^{*}=I_{n}$ for all $g$ in $G_{0}$. Let us show the reverse inclusion. For this purpose, let $\mathbf{H}$ be the division ring of quaternions with generators $1, \sqrt{-1}, j$, and $(\sqrt{-1}) j$. The space $\mathbf{H}^{1 \times k}$ of all $1 \times k$ matrices over $\mathbf{H}$ may be considered as a complex vector space which is identified to $\mathbf{C}^{1 \times 2 k}$ via the mapping

$$
\begin{array}{r}
\theta:\left[x_{1}+x_{k+1} j, \ldots, x_{k}+x_{2 k} j\right] \rightarrow\left[x_{1}, x_{2}, \ldots, x_{2 k}\right] \\
\left(x_{i} \in \mathbf{C}, 1 \leqslant i \leqslant 2 k\right) .
\end{array}
$$

Let $G_{0}$ act on $C^{1 \times 2 k}$ by right multiplication, then under the above identification $\theta$ there exists a bijection between $G_{0}$ and the group of all matrices of order $k$ over $\mathbf{H}$ which acts on $\mathbf{H}^{1 \times k}$ and preserves the symplectic product

$$
\left(\left[q_{1}, \ldots, q_{k}\right] \mid\left[q_{1}^{\prime}, \ldots, q_{k}^{\prime}\right]\right)=\sum_{i=1, \ldots, k} q_{i}\left(q_{i}^{\prime}\right)^{\vee} \quad\left(q_{i}, q_{i}^{\prime} \in \mathbf{H}\right)
$$

Now, suppose $X \in E$ with $X s_{k} X^{t}=0$ and $X X^{*}=I_{n}$, and let $x_{i}(1 \leqslant i \leqslant n)$ denote the $i$ th row of $X$; then it is easy to verify that $\left\{\theta^{-1}\left(x_{1}\right), \ldots, \theta^{-1}\left(x_{n}\right)\right\}$ forms an orthonormal system with respect to the above symplectic product. This orthonormal system may be completed to an orthonormal basis. Let $\sigma^{-1}$ denote the $k \times k$ symplectic matrix formed by this orthonormal basis with $n$ first rows consisting of $\theta^{-1}\left(x_{1}\right), \ldots, \theta^{-1}\left(x_{n}\right)$. Clearly, 


$$
\left[\begin{array}{c}
\theta^{-1}\left(x_{1}\right) \\
\vdots \\
\theta^{-1}\left(x_{n}\right)
\end{array}\right] \sigma=\overbrace{\left[I_{n} 0\right]}^{k} .
$$

Let $g_{0}$ denote the matrix of $G_{0}$ which corresponds to $\sigma$; then obviously $X g_{0}=1$. Hence, our contention is proved. Set $S_{\mathrm{H}}^{n, k}=\left\{X \in E: X s_{k} X^{t}=\right.$ 0 and $X X^{*}=I_{n}$; ; then we just showed that $S_{\mathrm{H}}^{n, k}$ is diffeomorphically identified to $K_{0} \backslash G_{0}$. For this reason, we shall call $S_{\mathrm{H}}^{n, k}$ the $(n, k)$-symplectic Stiefel manifold. Let $H\left(S_{\mathrm{H}}^{n, k}\right)$ (resp. $H\left(S_{\mathrm{H}}^{n, k}, \xi\right)$ ) be the algebra of functions on $S_{\mathrm{H}}^{n, k}$ which are obtained by restricting elements of $H\left(E^{*}\right)$ (resp. $H\left(E^{*}, \xi\right)$ ) to $S_{\mathrm{H}}^{n, k}$. By analogy with the notion of generalized spherical harmonics given in [6], elements of $H\left(S_{\mathbf{H}}^{n, k}\right)$ will be called symplectic Stiefel harmonics. Now, since $G_{0}$ acts transitively on $S_{\mathrm{H}}^{n, k}$, by Proposition 4.1 in [6], the restriction mapping $f \rightarrow f \mid S_{\mathrm{H}}^{n, k}\left(f \in H\left(E^{*}\right)\right)$ is a $G_{0}$-module isomorphism. On the other hand, according to the well-known "unitarian trick" (cf. [8]) the representation $R(\cdot, \xi)$ (of $G$ on $H(E, \xi)$ ) remains irreducible when restricted to $G_{0}$. As an immediate consequence of the above facts we have the following result.

THEOREM 2.3. If $R_{0}(\cdot, \xi)$ denotes the representation of $G_{0}$ which is obtained by right translation on $H\left(S_{\mathrm{H}}^{n, k}, \xi\right)$, then $R_{0}(\cdot, \xi)$ is irreducible. Moreover, its highest weight is indexed by $\left(m_{1}, \ldots, m_{n}, 0, \ldots, 0\right)(k$ factors $)$.

We conclude this article by singling out the following interesting problem: How to decompose explicitly the $G$-module $H\left(E^{*}\right)$ into simple $G$-modules. One general technique to obtain this decomposition consists of studying the decomposition of the Hilbert space $L^{2}\left(S_{\mathbf{H}}^{n, k}\right)$ in connection with the symplectic Stiefel harmonics (cf. [6]). This leads to the natural conjecture that $L^{2}\left(S_{\mathbf{H}}^{n, k}\right)$ is the Hilbert sum of the finite-dimensional Hilbert spaces $L^{2}\left(S_{\mathbf{H}}^{n, k}, \xi\right)$ where each $L^{2}\left(S_{\mathbf{H}}^{n, k}, \xi\right)$ is a (noncanonical) direct sum of $d_{\xi}$ copies of $H\left(S_{\mathbf{H}}^{n, k}, \xi\right), d_{\xi}$ being an integer determined by $\xi=\xi\left(m_{1}, \ldots, m_{n}\right)$. An alternate approach to solve this problem may be based on the so-called "algebraic Frobenius reciprocity" (cf. [4]). We shall give below a partial result following this second method (cf. [4] for the terminology used here).

Suppose that $k>n$ and $n$ is even, i.e., $n=2 l$. Let

$$
Z=\left[\begin{array}{cccc}
I_{l} & 0 & 0 & 0 \\
0 & 0 & I_{l} & 0
\end{array}\right] \in E
$$

and $\operatorname{Orb}(Z)=\{Z g: g \in G\}$. Then

$$
\operatorname{Orb}(Z)=\left\{X \in E: X s_{k} X^{t}=s_{l}, s_{l}=\left[\begin{array}{cc}
0 & -I_{l} \\
I_{l} & 0
\end{array}\right]\right\}
$$


and $\operatorname{Orb}(Z)$ may be diffeomorphically identified to the homogeneous space $K \backslash G$ where $K$ is the image of $\operatorname{Sp}(k-l, C)$ under the imbedding $\operatorname{Sp}(k-l, C)$ $\hookrightarrow \operatorname{Sp}(k, C)$. Let $S(\operatorname{Orb}(Z))$ denote the ring of functions on $\operatorname{Orb}(Z)$ obtained by restricting elements of $S\left(E^{*}\right)$ to $\operatorname{Orb}(Z)$.

THEOREM 2.4. If $\Re(\operatorname{Orb}(Z))$ denotes the ring of all rational functions on $\operatorname{Orb}(Z)$ then the restriction mapping $f \rightarrow f \mid \operatorname{Orb}(Z)\left(f \in H\left(E^{*}\right)\right)$ is a G-module isomorphism of $H\left(E^{*}\right)$ onto $\Re[\mathrm{Orb}(Z)]$.

Proof. Since $Z$ is quasi-regular the restriction mapping $f \rightarrow f \mid \operatorname{Orb}(Z)$ is a $G$-module isomorphism of $H\left(E^{*}\right)$ onto $S(\operatorname{Orb}(Z)$ ) (cf. [4, Proposition 5]). Now $\operatorname{Orb}(Z)$ is a smooth, hence normal, variety (cf. [5, Theorem 1, p. 93]); it follows then from [4, Proposition 9] that $R[\operatorname{Orb}(Z)]=S(\operatorname{Orb}(Z))$.

REMARK 2.5. Since the $G$-module structure of $R[\operatorname{Orb}(Z)]$ is in theory completely determined (cf. [4, Proposition 8]), Theorem 2.4 may be used to obtain a decomposition of the $G$-module $H\left(E^{*}\right)$ into simple $G$-submodules.

\section{REFERENCES}

1. A. Borel and A. Weil, Séminaire Bourbaki, 6ieme année: 1953/54, Exposé 100 par J. P. Serre: Représentations linéaires et espaces homogènes kählériens des groupes de Lie compacts, 2nd cor. ed., Secrétariat mathématique, Paris, 1959. MR 28 \#1087.

2. S. S. Gelbart, A theory of Stiefel harmonics, Trans. Amer. Math. Soc. 192 (1974), 29-50.

3. S. Helgason, Invariants and fundamental functions, Acta Math. 109 (1963), 241-258. MR 29 \#3581.

4. B. Kostant, Lie group representations on polynomial rings, Amer. J. Math. 85 (1963), 327-404. MR 28 \# 1252.

5. I. R. Safarevič, Foundations of algebraic geometry, Uspehi Mat. Nauk 24 (1969), no. 6(150), 3-184 = Russian Math. Surveys 24 (1969), no. 6, 1-178. MR 42 \#266.

6. T. Ton-That, Lie group representations and harmonic polynomials of a matrix variable, Trans. Amer. Math. Soc. 216 (1976), 1-46.

7. On holomorphic representations of symplectic groups, Bull. Amer. Math. Soc. 81 (1975), no. 6, 1069-1072.

8. H. Weyl, The classical groups. Their invariants and representations, Princeton Univ. Press, Princeton, N.J., 1939. MR 1, 42.

9. D. P. Żelobenko, Compact Lie groups and their representations, "Nauka", Moscow, 1970; English transl., Transl. Math. Monographs, vol. 40, Amer. Math. Soc., Providence, R.I., 1973.

Department of Mathematics, University of Iowa, Iowa City, Iowa 52242 